\title{
Discriminating phenotypic signatures identified for tocilizumab, adalimumab, and tofacitinib monotherapy and their combinations with methotrexate
}

\author{
Alison O'Mahony ${ }^{1 *}\left(\mathbb{D}\right.$, Markus R. John², Hannah Cho ${ }^{1}$, Misato Hashizume ${ }^{2}$ and Ernest H. Choy ${ }^{3}$
}

\begin{abstract}
Background: Clinical trials have shown combinations of anti-tumor necrosis factor biologicals plus methotrexate (MTX) are more effective treatments for rheumatoid arthritis than biological monotherapies, based, in part, on the assumption that MTX reduces the immunogenicity of biologicals. However, co-treatment with the anti-interleukin-6 receptor-alpha antibody tocilizumab (TCZ) and MTX does not demonstrate the same level of incremental benefit over TCZ monotherapy. Using the human primary cell based BioMAP phenotypic profiling platform, we investigated the impact of TCZ, adalimumab (ADA), and the small molecule drug tofacitinib (TOF), alone and in combination with MTX, on translational biomarkers that could indicate unique pharmacodynamic interactions outside those of reduced immunogenicity.
\end{abstract}

Methods: TCZ, ADA, and TOF, alone and in combination with MTX, were profiled in BioMAP systems at concentrations close to clinical exposure levels:TCZ, $200 \mu \mathrm{g} / \mathrm{ml}$; TOF1, $1.1 \mu \mathrm{M}$; TOF2, $0.12 \mu \mathrm{M}$; MTX, $10 \mu \mathrm{M}$. Changes in biomarkers were evaluated by statistical methods to determine whether combinations differed from the individual agents.

Results: Although the BioMAP activity profile for TCZ + MTX was not significantly different from that for TCZ alone, profiles for ADA + MTX and TOF1 + MTX or TOF2 + MTX had a greater number of statistically significant different activities $(P<0.01)$ than did agents profiled individually.

Conclusions: These data support the comparable efficacy of TCZ as monotherapy and as combination therapy and suggest that TOF, like ADA, may be more beneficial in combination with MTX. Taking an orthogonal approach to directly compare monotherapy and combination therapies indicates that MTX contributes to the efficacy of some, but not all, RA therapies and can be affected by factors additional to reduced immunogenicity.

Keywords: Inflammation, Biological therapy, Disease-modifying antirheumatic drugs, Rheumatoid arthritis, Ligands

\section{Background}

Methotrexate (MTX) is the first-line disease-modifying anti-rheumatic drug (DMARD) for the treatment of rheumatoid arthritis (RA). However, because disease control is not maintained for most patients, the American College of Rheumatology [1] and the European

\footnotetext{
*Correspondence: alisonomahony@eurofinsus.com

1 BioMAP Division, Eurofins DiscoverX, 310 Utah Avenue, South San

Francisco, CA 94080, USA

Full list of author information is available at the end of the article
}

League Against Rheumatism [2] recommend co-treating with synthetic or biological DMARDs. Given the scarcity of trials comparing DMARD regimens, the selection of combination drugs largely reflects physician preference rather than an evidence-based rationale. MTX is reported to reduce the potentially neutralizing immunogenicity of tumor necrosis factor-alpha (TNF) inhibitors (TNFis) [3], which may explain the superiority of TNFi + MTX over TNFi monotherapy [4, 5]. MTX + adalimumab (ADA) [6] and MTX + etanercept [7] clinical trials, however, showed similar additive benefit compared with either 
monotherapy despite the greater immunogenicity of ADA over etanercept, suggesting that reducing immunogenicity does not fully account for the enhanced efficacy of TNFi + MTX. Furthermore, combining MTX with the anti-interleukin (IL)-6 receptor-alpha (IL-6R $\alpha$ ) monoclonal antibody tocilizumab (TCZ) did not confer additive benefit above TCZ monotherapy $[8,9]$. Recently, oral Janus kinase (JAK) inhibitors such as TOF were developed or approved for RA [10]. Clinical trials have studied JAK combination therapy with MTX [11-15], though the scientific rationale is unclear given that these agents are nonimmunogenic. We hypothesized that additional pharmacodynamic (PD) interactions between MTX and biological DMARDs contribute to the additive therapeutic benefit for MTX and TNFi and that the comparable efficacy between MTX + TCZ and TCZ monotherapy might be due to more PD overlap and fewer additive interactions than MTX + TNFi.

These hypotheses were tested using the in vitro BioMAP phenotypic profiling platform (Eurofins DiscoverX, South San Francisco, CA) involving standardized and validated [16-19] multiplex human primary cell-based assays and a broad panel of translational biomarkers. This platform enables unbiased test agent characterization by detecting changes on the level of translational biomarkers across a broad set of assay systems modeling different human tissue and disease states. BioMAP assay systems are constructed with pooled healthy human donor primary cell co-cultures stimulated with cytokines or growth factors to recapitulate signaling networks relevant to human tissue or disease states [17, 20-25]. Each test agent/drug generates a signature activity profile reflecting changes in protein biomarker readouts relative to the vehicle control-treated systems. Biomarkers, including cell surface receptors, cytokines, chemokines, matrix molecules, and enzymes, within individual system environments are selected for therapeutic and biological relevance and validated using agents with known mechanisms of action (MoAs). Compound-mediated effects are quantified using immunoassays and, along with proliferation and viability assays, are predictive for disease outcomes or drug effects [17, 20-25].

Drug combinations may act either in an interactive manner on a common pathway(s) or in parallel with each other, with both agents acting independently. Such biological interactions could be promising with additive or synergistic activities reflected in altered biomarkers, discouraging if activities are reversed, or neutral if the drug combination produces activities identical to either agent or to both agents combined. Thus, taking an orthogonal approach to assess whether drug combinations meet a minimal requirement for the resultant effect of the combination to differ significantly from the monotherapies can inform on the potential for more efficacious therapy. Further, testing new agents, alone or in combination, in predictive [16] human disease models can support the identification, characterization, optimization, and application of new therapeutic strategies. Few DMARDs and even fewer of their combinations have been evaluated in such complex and reproducible human biological systems with multiple hierarchical levels of interaction networks connecting molecular targets, pathways, cells, and tissue. Such relationships are largely unknown, potentially masking important regulatory and feedback mechanisms that are often not evaluated before human studies are conducted.

BioMAP profiling was used in this analysis to predict whether the phenotypic impact of ADA, TCZ, and TOF monotherapy is modulated by co-treatment with MTX, specifically examining whether combination profiles were statistically significantly different from those of individual agents and identifying synergistic or beneficial outcomes and potential cytotoxicity.

\section{Methods \\ Cell culture}

A broad panel of BioMAP systems was used to generate phenotypic activity profiles of ADA, TCZ, and TOF, alone or in combination with MTX $[10,11,13]$. Biomarker levels of cell-associated and cell-membrane targets were measured using direct enzyme-linked immunosorbent assay (ELISA). An expanded biomarker readout panel was added to each system (Additional file 1: Table S1). Soluble factors from supernatants were quantified using either HTRF (CisBio, Bedford, MA) detection, beadbased multiplex immunoassay, or capture ELISA. Overt adverse effects on cell proliferation and viability (cytotoxicity) were measured by sulforhodamine B (SRB; adherent cells) and AlamarBlue (ThermoFisher Scientific, Waltham, MA) staining (suspension cells). For proliferation assays, individual cell types were cultured at subconfluence and measured at time points optimized for each system (48-96 h), as reported [20, 21, 23-26].

\section{Agents}

Test agents prepared in dimethyl sulfoxide (DMSO; small molecules, final concentration $\leq 0.1 \%$ ) or phosphatebuffered saline (biologicals) were added $1 \mathrm{~h}$ before stimulation and remained in culture for 24-144 h. Each plate contained drug (e.g., colchicine $1.1 \mu \mathrm{M})$, negative controls (nonstimulated conditions) and vehicle controls (e.g., $0.1 \%$ DMSO) appropriate for each system. TCZ was provided by F. Hoffmann-La Roche (Basel, Switzerland), and TOF and ADA were purchased from Selleckchem (Houston, TX) and Myoderm (Norristown, PA), respectively. Agents 
(See figure on next page.)

Fig. 1 BiOMAP profiles. a BioMAP profiles for all agents, $\mathbf{b}$ individual profile for tocilizumab, c clustering analysis of all agents at all concentrations, and $\mathbf{d}$ clustering analysis of other inhibitors (infliximab, etanercept, certolizumab, ruxolitinib, tofacitinib, and baricitinib)

were profiled alone and combined with MTX (Cayman Chemicals Inc., Ann Arbor, MI) in BioMAP systems at concentrations reflecting human therapeutic exposure or correlated with clinical exposure: TCZ, $200 \mu \mathrm{g} / \mathrm{ml}$; TOF, 1.1 and $0.12 \mu \mathrm{M}$; ADA, $200 \mu \mathrm{g} / \mathrm{ml}$; MTX, $10 \mu \mathrm{M}$. Assay readouts were measured after $48 \mathrm{~h}$ for the MyoF and HDFSAg systems and $72 \mathrm{~h}$ for the BT (B cells and T cells) system. Longer times were used for secreted immunoglobulin $G$ (sIgG) and proliferation end points in respective systems. Each readout parameter had three samples per data point, and each plate used a drug control in triplicate wells and eight vehicle control wells.

\section{Statistical analysis}

Statistical methods have been described [25, 26]. Measurements for each biomarker readout were divided by the mean value from DMSO controls to generate a ratio, which was then $\log _{10}$ transformed. Significance prediction envelopes were generated from biomarker levels for historical DMSO vehicle controls (95\% confidence interval). Key activities were annotated when two or more consecutive concentrations changed in the same direction relative to vehicle controls, were outside the significance envelope, and had an effect size $>10 \%\left(\mid \log _{10}\right.$ ratio $\left.\mid>0.05\right)$. Biomarker readouts were annotated as "modulated" if they increased in some system(s) and decreased in others. Pairwise correlation analysis was used to project the "proximity" of agent profiles from multidimensional space into two dimensions. Compounds and concentrations were represented by dots of different colors and sizes. Similar profiles (Pearson correlation coefficient $[r] \geq 0.7$ ) are connected by lines, and agents that did not cluster together were interpreted as mechanistically distinct [26].

To differentiate between agents profiled alone or combined with MTX, three statistical approaches were used to identify significant differences (hit scores): (a) P value hitunpaired $t$ test between combination versus single-agent profiles, where $\mathrm{P}<0.01$; (b) delta score hit-difference in raw optical density or median fluorescence intensity values (m), based on the formula $\Delta=|\mathrm{m} 1-\mathrm{m} 2| /|\mathrm{m} 1+\mathrm{m} 2|$, where $\Delta$ score hit is $>0.4$; (c) envelope hit-log ratio value is outside the historical DMSO vehicle control (95\% significance) envelope (gray zone) or $>6 \times$ standard deviation where no envelope exists (e.g., multiplex readouts). Hit scores were ranked (1-6) based on the following criteria: $1=\mathrm{P}$ value hit + delta score hit + activity outside the $95 \%$ vehicle control envelope; $2=$ P value hit (not delta score hit) + outside the $95 \%$ vehicle control envelope; $3=$ delta score hit (not
P value hit) but not outside the $95 \%$ vehicle control envelope; $4=\mathrm{P}$ value hit but not outside the $95 \%$ vehicle control envelope; $5=\mathrm{P}$ value significance or delta score hit not reached but outside the $95 \%$ vehicle control envelope; $6=$ no significant differences. Ranking scores $(1-6)$ were used to quantify the overall differences for combinations versus individual agents using BioMAP Viewer and Excel programs.

\section{Results \\ BioMAP profiling of MTX, ADA, TCZ, and TOF}

MTX, ADA, TCZ, and TOF were tested at a range of concentrations to evaluate changes in the levels of multiple translational biomarkers in the BioMAP Diversity PLUS Panel with two additional systems to generate a compoundspecific activity profile. As Fig. 1a shows, an overlay of the profiles for MTX, ADA, TCZ, and TOF indicates distinct phenotypic profiles for each agent at published plasma levels up to maximum concentrations consistent with their unique biological effects and MoAs. Analysis of the TCZ BioMAP profile (Fig. 1b) demonstrated TCZ-mediated inhibition of inflammation-related activities, including decreased P-selectin (4H), E-selectin (E-sel; HPNo), TNF- $\alpha$ (BT), IFN- $\gamma$-induced protein-10 (IP-10/CXCL10), IL-17A and IL-10 (HDFSAg), and CD69 (LPS). Decreased numbers of cytokines and chemokines in HDFSAg and BT systems indicate that $\mathrm{TCZ}$ primarily inhibits $\mathrm{T}$ cell and B-cell activation responses but has minimal impact on monocyte and macrophage responses in the LPS and $l$ Mphg systems, respectively. In contrast, ADA was active in multiple BioMAP systems across all doses (Supplementary Figure S1A). ADA exhibited potent anti-inflammatory effects in the LPS and $l$ Mphg systems consistent with its MoA, including decreased leukocyte recruitment and adhesion markers (vascular cell adhesion molecule-1 [VCAM-1], IL-8, intracellular cell adhesion molecule-1, E-sel), inhibition of immune activation markers (CD38, CD40, CD69, IP-10), decreased cytokine and chemokine production (IL-17A, IL-17F, IL-2, IL-1, IL-6, monocyte chemoattractant protein-1 [MCP-1/CCL2]) and monokine induced by IFN- $\gamma$ ([MIG/CXCL9]). Interestingly, an ADA-mediated increase in soluble interleukin (sIL)-6 levels was observed in the BT system. Modulation of matrix and tissue remodeling parameters such as increased collagens I-III in HDF3CGF and decreased matrix metalloproteinases- 1 and -9 in BE3C were also observed. TOF exhibited dose-dependent patterns of activity with high concentrations $(10 \mu \mathrm{M})$ and was broadly active in multiple systems, consistent with 

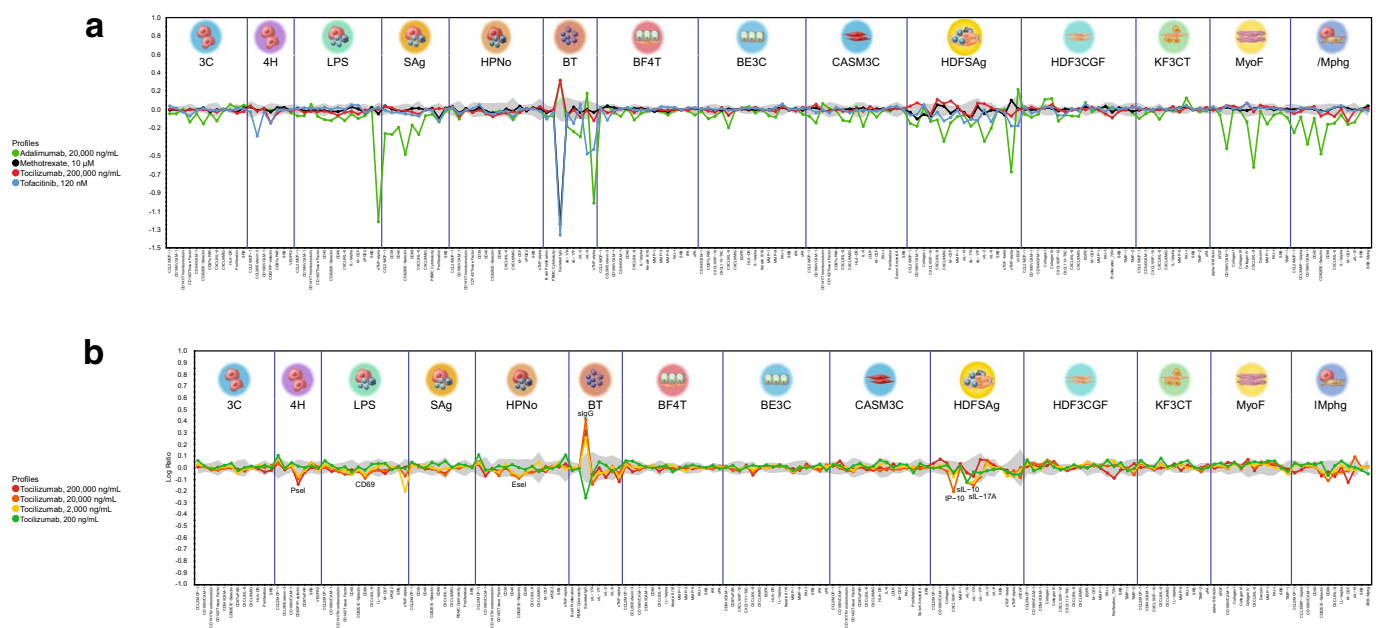

c

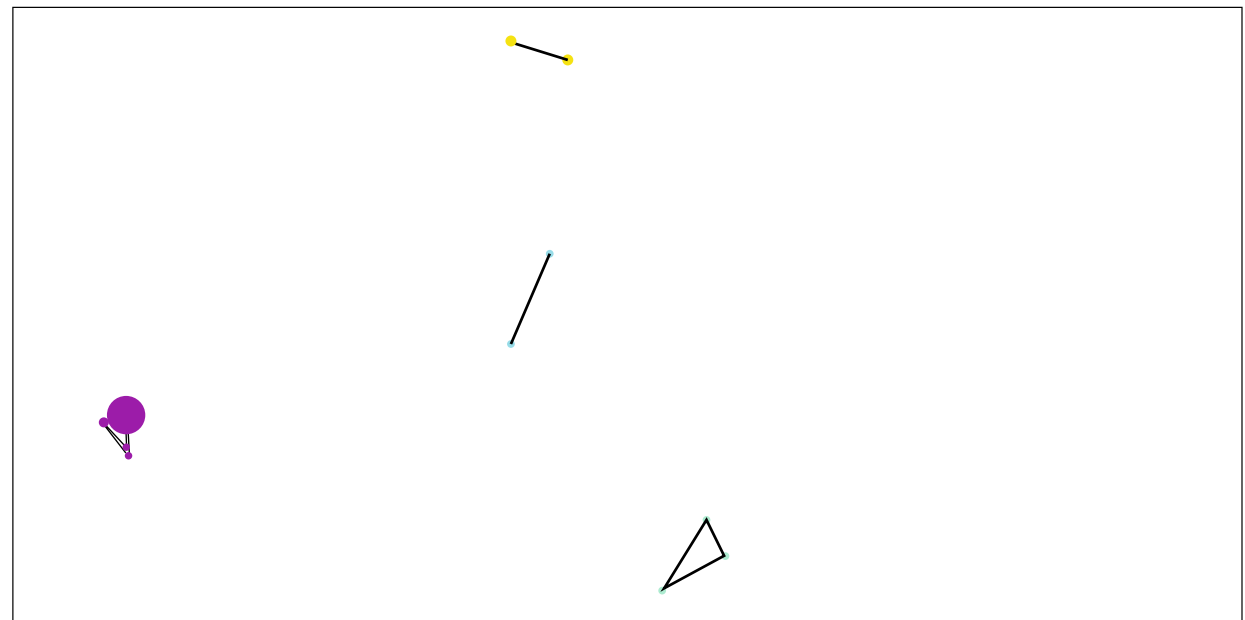

Adalimumab

Methotrexate

Tocilizumab

Tofacitinib

d

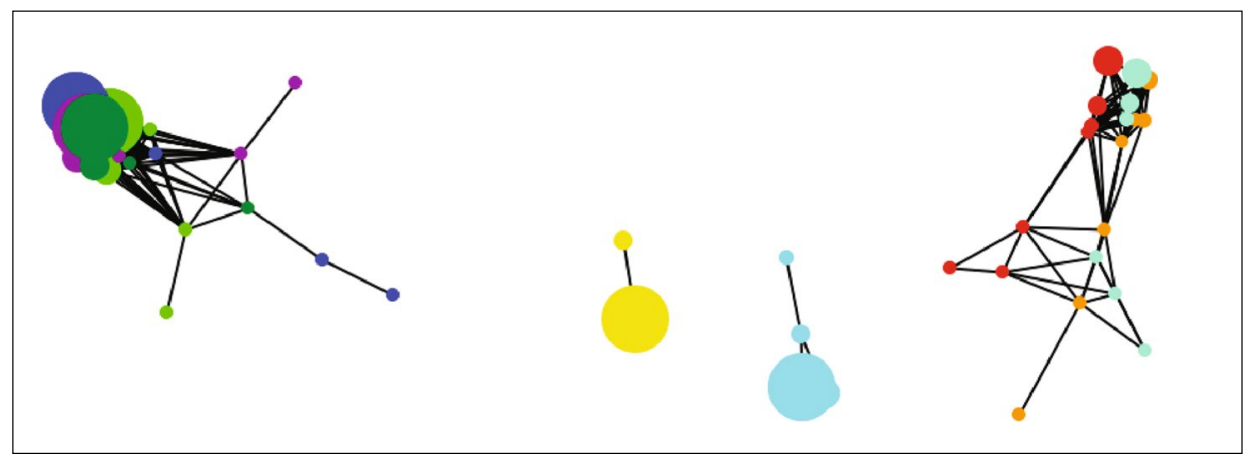

Adalimumab

Baricitinib

Certolizumab

Etanercept

Infliximab

Methotrexate

Ruxolitinib

Tocilizumab

Tofacitinib 
pan-JAK inhibition (Additional file 1: Figure S1B). A more selective pattern was observed at a lower, more clinically relevant concentration $(120 \mathrm{nM})$, consistent with inhibition of cytokine signaling through JAK1/JAK3 $\left(\mathrm{C}_{\max } 50 \mathrm{ng} /\right.$ $\mathrm{ml}=160 \mathrm{nM}$ at $5 \mathrm{mg}$ twice a day). Specifically, at lower exposures, TOF inhibited inflammation-related biomarker expression in multiple systems, modeling innate and adaptive immune responses including reduced eotaxin-3 and P-selectin (4H), CD69 (SAg), VCAM-1, MIG, IP-10 (CASMC and HDFSAg), sIL-6, TNF- $\alpha$, sIgG in BT and sIL10 and sIL-17 in the HDFSAg system modeling an inflamed RA joint. Additional vascular effects included decreased tissue factor $(3 \mathrm{C})$ and soluble vascular endothelial growth factor (HDFSAg).

MTX at the therapeutic exposure level of $10 \mu \mathrm{M}$ was uniquely active, with activities that included strong inhibition of IgG production (BT) and T-cell proliferation (SAg) along with modest inhibition of VCAM-1 in the HDFSAg system (Supplementary Figure S1C). MTX, like $\mathrm{TCZ}$, is more selectively active on T- and B-cell activation responses, whereas ADA and TOF are more broadly inhibitory on monocyte, macrophage, and T-cell activation responses and on fibroblast-related matrix modulation and tissue remodeling biology.

\section{Similarity clustering}

The activity profiles of each agent at all concentrations over the 14 different BioMAP systems were compared using Pearson correlation values for pairwise comparisons to generate function similarity maps (clustering analysis). TCZ, TOF, MTX, and ADA clustered within their own dose ranges but were distinct from each other (Fig. 1c). Similar analysis with other MoA-related agents from the BioMAP database (TNFis: infliximab [IFX], etanercept [ETN], and certolizumab [CTZ]; JAKis: baracitinib [BAR] and ruxolitinib [RUX]) showed that TCZ and MTX had unique profiles and did not cluster with other agents. ADA and TOF clustered at all concentrations within the corresponding mechanism class at $r>0.8$ (Fig. 1D).

Together these data confirm that the four test agentsTCZ, ADA, TOF, and MTX-exhibit distinctive phenotypic signatures reflecting diverse anti-inflammatory impact based on their distinct MoAs.

\section{Impact on sIL-6R trans-signaling-mediated inflammation biology}

Addition of soluble interleukin-6 receptor (sIL-6R) to BioMAP systems facilitated endogenous IL-6 transsignaling and modulation of several inflammatory effects in BioMAP systems, consistent with previous reports [27]. Eighteen activities were modulated by sIL$6 \mathrm{R} \alpha$ (Fig. 2a), which thus served as sentinel effects to evaluate the impact of MTX, ADA, TCZ, and TOF on IL-6 trans-signaling.

Figure $2 \mathrm{~b}-\mathrm{e}$ show the profiles for individual test agents, TCZ, TOF_0.1 $\mu \mathrm{M}$ (TOF2), TOF_1.1 $\mu \mathrm{M}$ (TOF1), and ADA overlaid with profiles for [agent + sIL-6R] and for sIL-6R $\alpha$ alone. Only TCZ fully reversed all key activities of sIL-6R $\alpha$ into the vehicle control envelope (18/18 [100\%]; Fig. 2b). In contrast, only some sIL-6R $\alpha$-mediated effects were reversed by ADA (5/18 [27\%]; Fig. 2c), TOF_0.1 (7/18 [38\%]; Fig. 2d), or TOF_1.1 (12/18 [66\%]; Fig. 2e). These data illustrate that TCZ was the only drug that effectively and comprehensively blocked IL-6-driven inflammation responses. After IL-6-IL-6R engagement, TOF blocks downstream JAK-mediated signals; however, it does not inhibit additional pathways mediated by IL-6elicited signaling kinases, including mitogen-activated protein kinase and phosphoinositide-3-kinase [28]. Similarly, though ADA and other TNFis block IL-6 production in response to TNF stimulation, they have little or no inhibitory effect on other inflammatory signals (e.g., IL-1 and TLR agonists) [29].

\section{MTX combinations of ADA versus TCZ versus TOF}

Anti-cancer strategies have used combination therapy to achieve new PD interactions that lead to enhanced efficacy and higher remission levels, but combinations can also yield more adverse effects. The MTX BioMAP profile (Additional file 1: Figure S1D) revealed that MTX strongly and selectively inhibited T-cell proliferation and B-cell IgG production. However, MTX $10 \mu \mathrm{M}$ had little or no effect on endothelial, fibroblast, or epithelial biology modeled in BioMAP systems. Conversely, ADA was broadly active across all BioMAP systems, impacting several stimulation-coupled responses in multiple cell types. This differential impact of MTX versus ADA is consistent with clinical observations [30] and supports combining MTX with biologicals [2, 6 , 7]. The combination of ADA + MTX revealed more statistically significant different activities $(\mathrm{P}<0.01$, paired $t$ test) than ADA and MTX profiled individually under standard conditions (9/29 hits, $31 \%)$ and to an even greater extent under additional sIL-6R $\alpha$-mediated stimulation (16/26 hits, $61 \%)$. Activities inhibited to a greater extent by ADA + MTX combinations than with ADA alone under both stimulation conditions included several anti-inflammatory markers, MCP-1 (BF4T), IL-8, macrophage colony-stimulating factor 1 (HDF3CGF), and VCAM-1 (lMphg). Moreover, several matrix-/tissue-remodeling effects such as basic fibroblast growth factor (bFGF), collagens I and IV, and fibroblast proliferation were significantly inhibited to a greater extent with ADA + MTX than with either agent alone. These data clearly illustrate that combination 


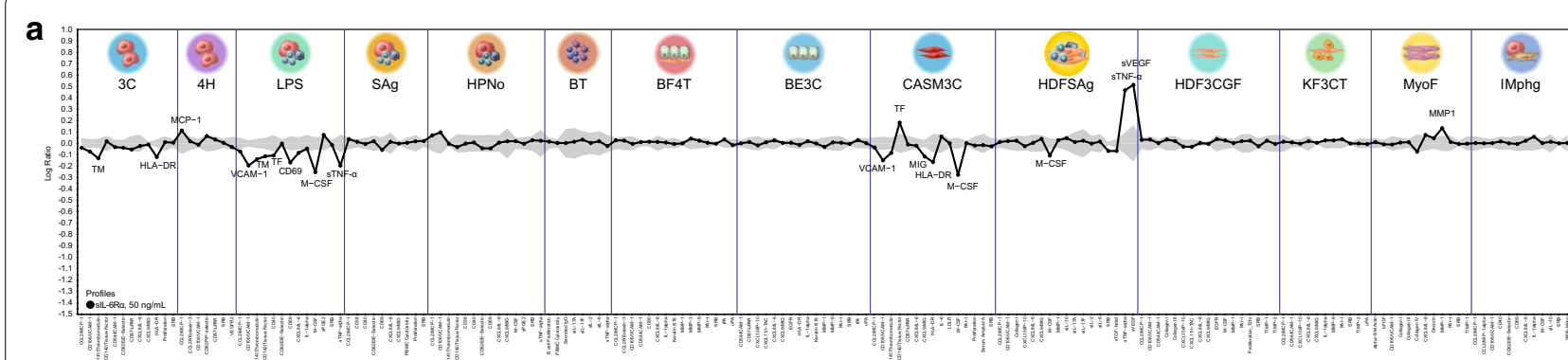

b

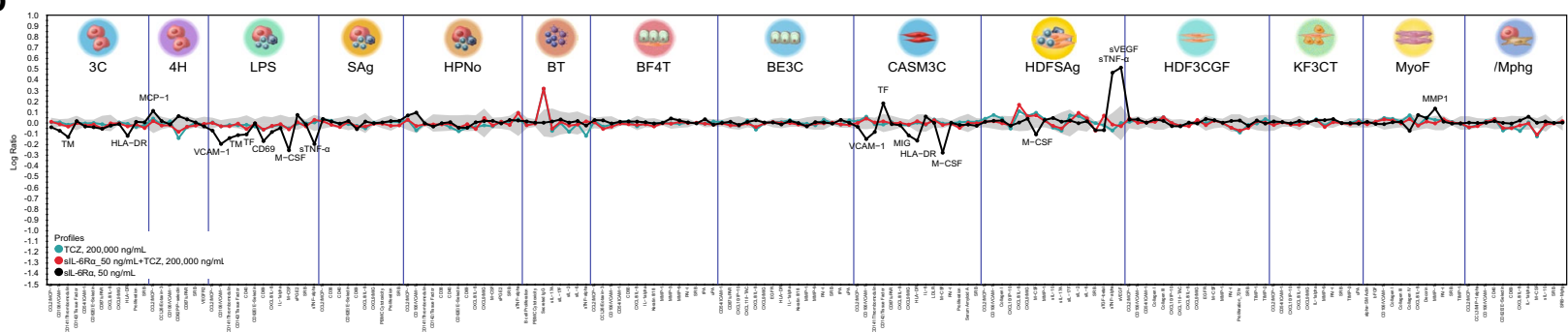

C

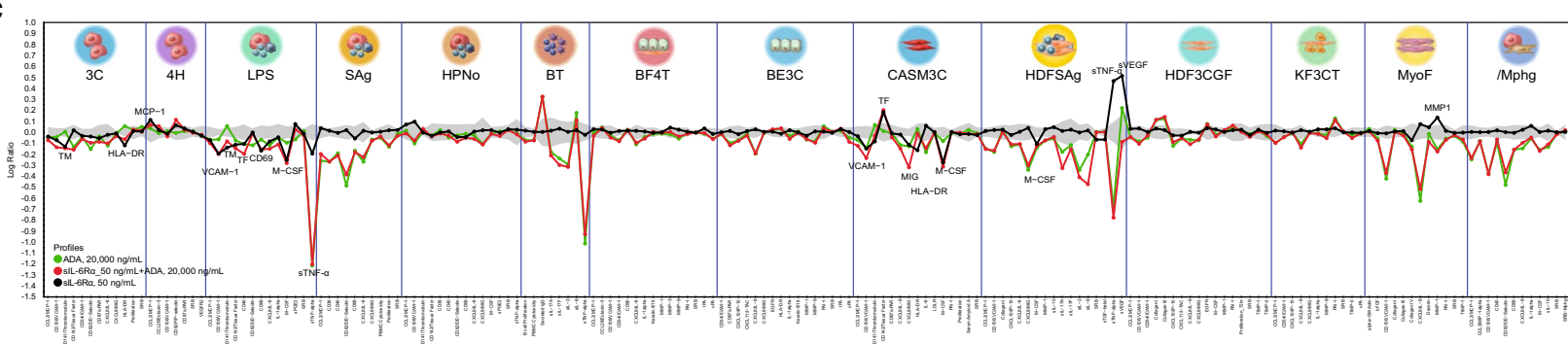

d
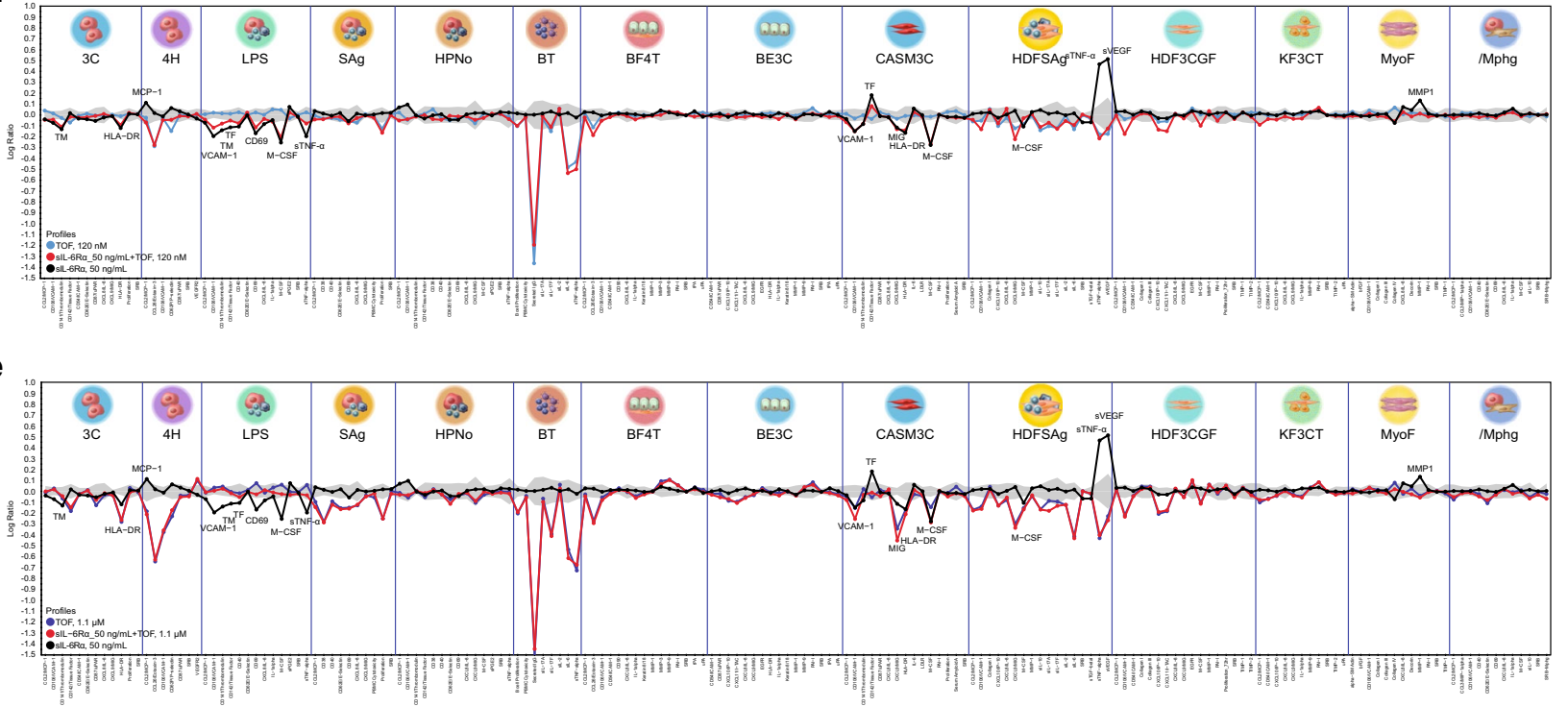

Fig. 2 Impact on inflammation. Impact of a all agents, b tocilizumab, c adalimumab, d tofacitinib_0.1, and e tofacitinib_1.1 on soluble IL-6 receptor trans-signaling-mediated inflammation biology 
ADA + MTX has statistically significantly more nonoverlapping effects on immune function, inflammation markers, and matrix-remodeling end points in primary human cell disease models than does either agent alone. In contrast, under standard stimulation conditions, $8 / 18$ hits (44\%) differentiated TCZ + MTX from TCZ alone. Notably, under sIL-6R stimulation conditions, only $1 / 16$ hits (6\%) was significantly different with TCZ + MTX than with TCZ alone. Indeed, in this IL-6-trans-signaling-driven environment, the only enhanced impact of TCZ + MTX combination compared with TCZ alone $(\mathrm{P}<0.01)$ was greater antiproliferative effects on endothelial cells (3C) and B cells (BT). $\mathrm{TCZ}+\mathrm{MTX}$ combination had more overlapping effects and less chance to silence or enhance the other agent's effects, especially in an IL-6-trans-signaling-driven inflammation environment, and did not elicit activities beyond enhanced antiproliferative effects compared with TCZ or MTX alone. However, MTX + ADA combination has more additive biological effects than $\mathrm{MTX}+\mathrm{TCZ}$ or the individual agents.

The study was expanded to include two concentrations of TOF, consistent with pan-JAK inhibition (TOF_1 tested at $1.1 \mu \mathrm{M}$; TOF1) and JAK1/3 inhibition (TOF_0.1 tested at $120 \mathrm{nM}$; TOF2) [31-33]. First, under standard stimulation conditions, $7 / 33$ hits (21\%) differentiated the TOF_1+MTX combination from TOF_1 alone. At the lower concentration of TOF (TOF_0.1; TOF2), 9/17 hits (53\%) differentiated the TOF_0.1+MTX combination from TOF_0.1 alone. Second, in the presence of sIL-6R $\alpha$ mediated trans-signaling, 22/42 hits (52\%) differentiated the TOF_1+MTX combination from TOF_1 alone, and $10 / 25$ hits (40\%) differentiated the TOF_0.1 + MTX combination from TOF_0.1 alone. Activities modulated by combinations of TOF $1+$ MTX or TOF $2+$ MTX under both standard and sIL-6R-mediated stimulation included cytokine and chemokine levels (macrophage and granulocyte colony-stimulating factors), inflammation markers (VCAM-1, E-sel, and IP-10), and tissueremodeling activities (thrombomodulin and plasminogen activator inhibitor 1). Combining TOF at clinical or supraclinical concentrations with MTX affects immune function, inflammation markers, and matrix-remodeling end points differently from using TOF or MTX alone.

Overall, these results show that though multiple effects of both ADA and TOF were significantly altered when combined with MTX, PD interactions between TCZ and MTX were significantly less pronounced in BioMAP systems. This is consistent with the comparable efficacies of TCZ monotherapy and combination therapy in clinical trials [9] and real life [8], suggesting that combining TOF with MTX may be more beneficial.

\section{Discussion}

Conventional synthetic DMARDs produce limited efficacy in RA, combination strategies are poorly understood from a mechanistic perspective, and optimized combinations have not been determined. The MoAs of conventional synthetic DMARDs in RA remain unknown, further undermining the ability to predict greater efficacy and to optimize dosing for combination strategies. Biological agents have improved the management and prognosis of RA, and five classes are licensed: TNFi, IL-1 inhibitor, B-cell depleter, T-cell costimulation blocker, and IL-6R $\alpha$ inhibitor. The chimeric TNFi infliximab was the first monoclonal antibody assessed in RA, and, though it was efficacious, many patients developed human antichimeric antibody responses associated with reduced efficacy and increased risk for infusion reaction. A randomized controlled trial demonstrated that concomitant MTX reduced the immunogenicity of infliximab [34], and subsequent studies found that combination therapy with MTX and ADA or etanercept was superior to biological monotherapy. Clinical trials investigating biological agents and novel DMARDs, including TOF clinical trials (despite the fact that TOF is nonimmunogenic), have since added active treatment or placebo to MTX. The benefits of combining MTX with a TNFi appear to arise preferentially from its anti-immunoglobulin effects, which also control autoantibodies against the drug, whereas the MTX + TOF combination results in a broader-spectrum regimen. Guidelines recommend combining biological agents with MTX to treat RA patients unless MTX is contraindicated or the patient has tolerability issues $[1,2]$. It is assumed that the added benefit of cotreating RA patients with a TNFi + MTX is associated with the ability of MTX to reduce the immunogenicity of the biological; however, the present profiling data introduce the compelling notion that these drugs have additional PD interactions that can lead to enhanced efficacy independent of dampened immunogenic response to the TNFi. ADA + MTX was significantly more active than either agent alone, illustrating that, in addition to reducing TNFi immunogenicity, this combination has a greater nonoverlapping impact on RA-related biology. The significantly altered results for TOF monotherapy compared with TOF + MTX further support this finding. Given that TOF is not a biological, the anti-immunogenic impact of MTX is not relevant, and the additional effects of the combination compared with TOF alone indicate significant additive effects for the combination, consistent with previous publications. In contrast, the TCZ + MTX combination was largely unchanged from the profile for $\mathrm{TCZ}$ alone, indicating limited therapeutic benefit for the combination, again consistent with previous observations from clinical trials $[8,9]$. Thus, in the absence of new 
PD interactions, the potential for enhanced or unique activities is lowered, and added clinical benefit may not materialize. In the ADACTA trial, TCZ monotherapy demonstrated superiority over ADA monotherapy [35]. Subgroup analysis revealed that patients who tested positive for anti-cyclic citrullinated peptide or rheumatoid factor obtained additional benefit from TCZ therapy [35]. The data presented here show that TNFis do not inhibit B-cell function in BioMAP assays. B cells are likely to play a role in driving inflammation in seropositive RA patients, rendering them less likely to benefit from TNFis than from TCZ.

A limitation of this study is that tolerability issues with MTX, such as nausea and hair loss, may not be modeled by BioMAP systems. In this context, it is interesting that TCZ demonstrates some MTX-like effects (making the use of MTX partially clinically redundant) without MTX-associated tolerability issues. However, the BioMAP system showed that MTX acts primarily on $\mathrm{T}$ and $\mathrm{B}$ cells, which are involved in RA pathogenesis and antibody production-effects that may be inhibited by TCZ. TNFis inhibited the innate immune response mediated largely by monocytes and macrophages but had minimal effect on T- and B-cell responses. This study has shown that differences exist between PD manifestations of biologics or small molecules in combination with MTX and the respective monotherapies. Further research using different methodologies and models would be needed to deconvolute the mechanisms underlying these differences and to interpret the effects with respect to outcomes in patients with RA.

\section{Conclusions}

Overall, these data show that though combination therapies may or may not alter the activities of small and large molecules, they have the potential to manifest unique PD interactions, potentially leading to a greater disease-modifying impact. The BioMAP evidence-based approach for preclinical testing of agents in combination may be useful to guide feasibility and dosing strategies and to assess, at a minimum, whether combination therapy is active, safe, and statistically biologically different from monotherapy with individual agents, but results must be confirmed in the heterogeneous RA patient population.

\section{Additional file}

Additional file 1: Table S1. Expanded soluble readout panel for each BioMAP system. Figure S1. BioMAP profiles.

\section{Abbreviations}

ADA: adalimumab; BAR: baracitinib; BT: B cell/T cell BioMAP system; CCL2: chemokine (C-C motif) ligand 2; CD: cluster of differentiation; CTZ: certolizumab; CXCL: C-X-C motif chemokine ligand; DMARD: disease-modifying antirheumatic drug; DMSO: dimethyl sulfoxide; ELISA: enzyme-linked immunosorbent assay; E-sel: E-selectin; ETN: etanercept; HDFSAg: human dermal fibroblasts + peripheral blood mononuclear cells BioMAP system; HTRF: homogeneous time-resolved fluorescence; IFN- $\gamma$ : interferon-gamma; IFX: infliximab; IgG: immunoglobulin G; IL: interleukin; IL-6Ra: interleukin-6 receptor-alpha; IP-10: induced protein-10; JAK: Janus kinase; LPS: lipopolysaccharide; MCP-1: monocyte chemoattractant protein-1; MIG: monokine induced by interferon-gamma; MoAs: mechanisms of action; MTX: methotrexate; MyoF: lung fibroblasts BioMAP system; PD: pharmacodynamic; RA: rheumatoid arthritis; RUX: ruxolitinib; SIgG: secreted immunoglobulin G; Sil: soluble interleukin; SRB: sulforhodamine B; TCZ: tocilizumab; TLR: Toll-like receptor; TNF/TNF-a: tumor necrosis factor-alpha; TNFi: tumor necrosis factor-alpha inhibitor; TOF: tofacitinib; VCAM-1: vascular cell adhesion molecule-1.

\section{Authors' contributions}

All authors were involved in drafting the article or revising it critically for important intellectual content. AOM had full access to all the data in the study and takes responsibility for the integrity of the data and the accuracy of the data analysis. Study conception and design: AOM, MRJ, EHC. Development of methodology: AOM. Acquisition of data: AOM, HC. Analysis and interpretation of data: $\mathrm{AOM}, \mathrm{HC}, \mathrm{MH}, \mathrm{EHC}$. Drafting and revising the manuscript critically for important intellectual content: all authors. All authors read and approved the final manuscript.

\section{Author details}

${ }^{1}$ BioMAP Division, Eurofins DiscoverX, 310 Utah Avenue, South San Francisco, CA 94080, USA. ${ }^{2}$ F. Hoffmann-La Roche AG, 4070 Basel, Switzerland. ${ }^{3}$ Division of Infection and Immunity, CREATE Centre, Cardiff University, Cardiff CF10 3AT, UK.

\section{Acknowledgements}

Dat Nguyen performed compound handling activities, Jennifer Melrose performed cell culture activities, and Ellen Berg conducted data review. All are employees of DiscoverX Corporation.

The first draft of the manuscript was prepared by Dr. O'Mahony. Revisions to the manuscript were made by all authors, with editorial assistance provided by ApotheCom (Yardley, PA) on behalf of F. Hoffmann-La Roche Ltd.

\section{Competing interests}

Alison O'Mahony has nothing to disclose. Markus R. John is an employee of and owns stock in Roche ( $>10,000)$. Hannah Cho has nothing to disclose. Misato Hashizume has nothing to disclose. Ernest $\mathrm{H}$. Choy has received a research grant from Roche $(>\$ 10,000)$.

\section{Availability of data and materials}

The data that support the findings of this study are available from the corresponding author on reasonable request and with permission from $\mathrm{F}$. Hoffmann-La Roche.

\section{Consent for publication}

Not applicable.

\section{Ethics approval and consent to participate}

Ethics review board approval and patient consent were not required for this study. Primary human cell lines were purchased commercially from vendors (HemaCare, Van Nuys, CA; Lonza, Walkersville, MD; StemCell Technologies, Vancouver, BC, Canada) who have their own ethics review boards and follow the American Association of Tissue Banks, Association of Organ Procurement Organizations, or Eye Bank Association of America ethical practices and informed consent guidelines. All studies followed the US guidelines for research on human subjects as defined by the US Department of Health and Human Services Protection of Human Subjects (45 CFR 46).

\section{Funding}

This work was supported by F. Hoffmann-La Roche Ltd. The sponsor was involved in the study design; collection, analysis, and interpretation of data; and manuscript revision. 


\section{Publisher's Note}

Springer Nature remains neutral with regard to jurisdictional claims in published maps and institutional affiliations.

Received: 27 March 2018 Accepted: 30 May 2018

Published online: 07 June 2018

\section{References}

1. Singh JA, Saag KG, Bridges SL Jr, AkI EA, Bannuru RR, Sullivan MC, et al. 2015 American College of Rheumatology Guideline for the Treatment of Rheumatoid Arthritis. Arthritis Care Res. 2016;68(1):1-25.

2. Smolen JS, Landewe R, Breedveld FC, Buch M, Burmester G, Dougados $M$, et al. EULAR recommendations for the management of rheumatoid arthritis with synthetic and biological disease-modifying antirheumatic drugs: 2013 update. Ann Rheum Dis. 2014:73(3):492-509.

3. Burmester GR, Kivitz AJ, Kupper H, Arulmani U, Florentinus S, Goss SL, et al. Efficacy and safety of ascending methotrexate dose in combination with adalimumab: the randomised CONCERTO trial. Ann Rheum Dis. 2015;74(6):1037-44.

4. Murdaca G, Spano F, Contatore M, Guastalla A, Penza E, Magnani O, et al. Immunogenicity of infliximab and adalimumab: what is its role in hypersensitivity and modulation of therapeutic efficacy and safety? Expert Opin Drug Saf. 2016;15(1):43-52

5. O'Dell JR. Rheumatoid arthritis. In: Stone JH, editor. A clinician's pearls and myths in rheumatology. New York: Springer; 2016. p. 1-13.

6. Breedveld FC, Weisman MH, Kavanaugh AF, Cohen SB, Pavelka K, van Vollenhoven $\mathrm{R}$, et al. The PREMIER study: a multicenter, randomized, double-blind clinical trial of combination therapy with adalimumab plus methotrexate versus methotrexate alone or adalimumab alone in patients with early, aggressive rheumatoid arthritis who had not had previous methotrexate treatment. Arthritis Rheum. 2006;54(1):26-37.

7. Klareskog L, Van Der Heijde D, de Jager JP, Gough A, Kalden J, Malaise $M$, et al. Therapeutic effect of the combination of etanercept and methotrexate compared with each treatment alone in patients with rheumatoid arthritis: double-blind randomised controlled trial. Lancet. 2004;363(9410):675-81.

8. Bykerk VP, Ostor AJ, Alvaro-Gracia J, Pavelka K, Román Ivorra JA, Graninger W, et al. Comparison of tocilizumab as monotherapy or with add-on disease-modifying antirheumatic drugs in patients with rheumatoid arthritis and inadequate responses to previous treatments: an open-label study close to clinical practice. Clin Rheumatol. 2015;34(3):563-71.

9. Dougados M, Kissel K, Sheeran T, Tak PP, Conaghan PG, Mola EM, et al. Adding tocilizumab or switching to tocilizumab monotherapy in methotrexate inadequate responders: 24-week symptomatic and structural results of a 2 year randomized controlled strategy trial in rheumatoid arthritis (ACT-RAY). Ann Rheum Dis. 2013;72(1):43-50.

10. XELJANZ (tofacitinib) tablets for oral administration. New York, NY: Pfizer Labs; 2012.

11. Burmester GR, Blanco R, Charles-Schoeman C, Wollenhaupt J, Zerbini C, Benda B, et al. Tofacitinib (CP-690,550) in combination with methotrexate in patients with active rheumatoid arthritis with an inadequate response to tumour necrosis factor inhibitors: a randomised phase 3 trial. Lancet. 2013;381(9865):451-60

12. Kremer J, Li ZG, Hall S, Fleischmann R, Genovese M, Martin-Mola E, et al. Tofacitinib in combination with nonbiologic disease-modifying antirheumatic drugs in patients with active rheumatoid arthritis: a randomized trial. Ann Intern Med. 2013;159(4):253-61.

13. Van Der Heijde D, Tanaka Y, Fleischmann R, Keystone E, Kremer J, Zerbini C, et al. Tofacitinib (CP-690,550) in patients with rheumatoid arthritis receiving methotrexate: twelve-month data from a twentyfour-month phase III randomized radiographic study. Arthritis Rheum. 2013;65(3):559-70.

14. Tanaka Y, Emoto K, Cai Z, Aoki T, Schlichting D, Rooney T, et al. Efficacy and safety of baricitinib in Japanese patients with active rheumatoid arthritis receiving background methotrexate therapy: a 12-week, double-blind, randomized placebo-controlled study. J Rheumatol. 2016;43(3):504-11.

15. Genovese MC, van Vollenhoven RF, Pacheco-Tena C, Zhang Y, Kinnman N. VX-509 (decernotinib), an oral selective JAK-3 inhibitor, in combination with methotrexate in patients with rheumatoid arthritis. Arthritis Rheumatol. 2016;68(1):46-55.

16. Berg EL, Yang J, Polokoff MA. Building predictive models for mechanismof-action classification from phenotypic assay data sets. J Biomol Screening. 2013;18(10):1260-9.

17. Berg EL, Kunkel EJ, Hytopoulos E, Plavec I. Characterization of compound mechanisms and secondary activities by BioMAP analysis. J Pharmacol Toxicol Methods. 2006;53(1):67-74.

18. Ciceri P, Muller S, O'Mahony A, Fedorov O, Filippakopoulos P, Hunt JP, et al Dual kinase-bromodomain inhibitors for rationally designed polypharmacology. Nat Chem Biol. 2014;10(4):305-12.

19. Berg EL, Kunkel EJ, Hytopoulos E. Biological complexity and drug discovery: a practical systems biology approach. Syst Biol. 2005;152(4):201-6.

20. Kunkel EJ, Dea M, Ebens A, Hytopoulos E, Melrose J, Nguyen D, et al. An integrative biology approach for analysis of drug action in models of human vascular inflammation. FASEB J. 2004;18(11):1279-81.

21. Kunkel EJ, Plavec I, Nguyen D, Melrose J, Rosler ES, Kao LT, et al. Rapid structure-activity and selectivity analysis of kinase inhibitors by BioMAP analysis in complex human primary cell-based models. Assay Drug Dev Tech. 2004:2(4):431-41.

22. Houck KA, Dix DJ, Judson RS, Kavlock RJ, Yang J, Berg EL. Profiling bioactivity of the ToxCast chemical library using BioMAP primary human cell systems. J Biomol Screen. 2009;14(9):1054-66.

23. Xu D, Kim Y, Postelnek J, Vu MD, Hu DQ, Liao C, et al. RN486, a selective Bruton's tyrosine kinase inhibitor, abrogates immune hypersensitivity responses and arthritis in rodents. J Pharmacol Exp Ther. 2012:341(1):90-103.

24. Bergamini G, Bell K, Shimamura S, Werner T, Cansfield A, Müller K, et al. A selective inhibitor reveals PI3Kgamma dependence of $\mathrm{T}(\mathrm{H}) 17$ cell differentiation. Nat Chem Biol. 2012;8(6):576-82.

25. Melton AC, Melrose J, Alajoki L, Privat S, Cho H, Brown N, et al. Regulation of IL-17A production is distinct from IL-17F in a primary human cell co-culture model of T cell-mediated B cell activation. PLOS ONE. 2013;8(3):e58966

26. Berg EL, Yang J, Melrose J, Nguyen D, Privat S, Rosler E, et al. Chemical target and pathway toxicity mechanisms defined in primary human cell systems. J Pharmacol Toxicol Methods. 2010;61(1):3-15.

27. Scheller J, Chalaris A, Schmidt-Arras D, Rose-John S. The pro- and antiinflammatory properties of the cytokine interleukin-6. Biochim Biophys Acta. 2011;1813(5):878-88.

28. Heinrich PC, Behrmann I, Haan S, Hermanns HM, Muller-Newen G, Schaper F. Principles of interleukin (IL)-6-type cytokine signalling and its regulation. Biochem J. 2003;374(pt 1):1-20.

29. Tanaka T, Kishimoto T. Targeting interleukin-6: all the way to treat autoimmune and inflammatory diseases. Int J Biol Sci. 2012;8(9):1227-36.

30. Shiozawa K, Tanaka Y, Yoshihara R, Imura S, Murata M, Yamane T, et al. Serum levels and pharmacodynamics of methotrexate and its metabolite 7-hydroxy methotrexate in Japanese patients with rheumatoid arthritis treated with 2-mg capsule of methotrexate three times per week. Mod Rheumatol. 2005;15(6):405-9.

31. Thoma $G$, Nuninger $F$, Falchetto $R$, Hermes $E$, Tavares $G A$, Vangrevelinghe $E$, et al. Identification of a potent Janus kinase 3 inhibitor with high selectivity within the Janus kinase family. J Med Chem. 2011;54(1):284-8.

32. Dymock BW, See CS. Inhibitors of JAK2 and JAK3: an update on the patent literature 2. Expert Opin Ther Patents. 2013;23(4):449-501.

33. Flanagan ME, Blumenkopf TA, Brissette WH, Brown MF, Casavant JM, Shang-Poa C, et al. Discovery of CP-690,550: a potent and selective Janus kinase (JAK) inhibitor for the treatment of autoimmune diseases and organ transplant rejection. J Med Chem. 2010;53(24):8468-84.

34. Maini RN, Breedveld FC, Kalden JR, Smolen JS, Furst D, Weisman MH, et al. Sustained improvement over two years in physical function, structural damage, and signs and symptoms among patients with rheumatoid arthritis treated with infliximab and methotrexate. Arthritis Rheum. 2004:50(4):1051-65.

35. Gabay C, Emery P, van Vollenhoven R, Dikranian A, Alten R, Pavelka K, et al. Tocilizumab monotherapy versus adalimumab monotherapy for treatment of rheumatoid arthritis (ADACTA): a randomised, double-blind, controlled phase 4 trial. Lancet. 2013:381:1541-50. 